Article

\title{
Long-Term Converse Magnetoelectric Response of Actuated 1-3 Multiferroic Composite Structures
}

\author{
Ryan Stampfli, Nha Uyen Huynh and George Youssef *D \\ Experimental Mechanics Laboratory, Mechanical Engineering Department, San Diego State University, \\ 5500 Campanile Drive, San Diego, CA 92182, USA; r.stampfli.me@gmail.com (R.S.); nhuynh2@sdsu.edu (N.U.H.) \\ * Correspondence: gyoussef@sdsu.edu
}

Citation: Stampfli, R.; Huynh, N.U.; Youssef, G. Long-Term Converse

Magnetoelectric Response of Actuated 1-3 Multiferroic Composite Structures. Magnetochemistry 2021, 7, 55. https://doi.org/10.3390/ magnetochemistry7040055

Academic Editor: Roman Boca

Received: 29 March 2021

Accepted: 16 April 2021

Published: 20 April 2021

Publisher's Note: MDPI stays neutral with regard to jurisdictional claims in published maps and institutional affiliations.

Copyright: (c) 2021 by the authors. Licensee MDPI, Basel, Switzerland. This article is an open access article distributed under the terms and conditions of the Creative Commons Attribution (CC BY) license (https:// creativecommons.org/licenses/by/ $4.0 /)$.

\begin{abstract}
Multiferroic composite materials operating under the principle of strain mediation across the interfaces separating different material boundaries address many limitations of single-phase magnetoelectric materials. Although significant research has been conducted to explore their responses relating to the topography and directionality of material polarization and magnetic loading, there remain unanswered questions regarding the long-term performance of these multiferroic structures. In this study, a multiferroic composite structure consisting of an inner Terfenol-D magnetostrictive cylinder and an outer lead zirconate titanate (PZT) piezoelectric cylinder was investigated. The composite was loaded over a 45-day period with an AC electric field $(20 \mathrm{kV} / \mathrm{m})$ at a near-resonant frequency $(32.5 \mathrm{kHz})$ and a simultaneously applied DC magnetic field of 500 Oe. The long-term magnetoelectric and thermal responses were continuously monitored, and an extensive micrographic analysis of pretest and post-test states was performed using scanning electron microscopy (SEM). The extended characterization revealed a significant degradation of $\approx 30-50 \%$ of the magnetoelectric response, whereas SEM micrographs indicated a reduction in the bonding interface quality. The increase in temperature at the onset of loading was associated with the induced oscillatory piezoelectric strain and accounted for $28 \%$ of the strain energy loss over nearly one hour.
\end{abstract}

Keywords: strain mediation; multiferroics; magnetoelectric coupling; long-term response; composite multiferroics

\section{Introduction}

Research interest in single and multiphase multiferroic materials has expanded greatly in the past two decades to reduce the footprint of electronic devices while efficiently managing power consumption. These research efforts are widely spread over multiple investigation areas that span from chemistry to material science and from mechanical engineering to device reliability testing to understand and optimize the underlying fundamental phenomena to improve the resulting magnetoelectric coupling metrics [1-4]. Multiferroics for magnetoelectric applications possess intrinsic or extrinsic coupling between two or more ferroic order parameters, wherefore they can bidirectionally convert electric energy to magnetic energy. When considered in the single-phase form, intrinsic multiferroics are uncommon and exhibit substantially reduced magnetoelectric coupling coefficients at room temperature, decisively restricting their integration in devices outside of laboratory conditions. In an engineering attempt to resolve these issues, multiferroic composite materials, consisting of separate ferroelectric and ferromagnetic material phases, can be created with a bidirectional coupling through charge, spin, or strain transfer across material interfaces [2,3,5-10]. In the strain-mediated approach, the magnetostriction in ferromagnetic materials and the piezoelectric effect in ferroelectric materials are exploited. The converse magnetoelectric effect (CME) is based on applying an electric field to the piezoelectric material, resulting in a change in the magnetization of the ferromagnetic material due to the mediation of strain. Therefore, the CME coefficient is the coupling 
efficacy metric between the applied and generated fields and is defined as the output magnetic flux ratio to the input voltage.

Although significant research effort has recently been dedicated to nanoscale investigations of composite multiferroics, experimental studies of concentric cylinder composites (1-3 composites [11]) remain relatively limited to the macroscale. The overall magnetoelectric response is then implicitly defined as a function of selecting the material phases, the geometry, and the quality and type of the interface between the different constituents. Chavez et al. investigated the CME response of concentric PZT/Terfenol-D composite cylinders with epoxy-bonded and shrink-fit interfaces to elucidate the effect of the interfaces [12]. A maximum CME coefficient of $525 \mathrm{mG} / \mathrm{V}$ was found for the epoxy-bonded interface, while the shrink-fit interface reached a mere $330 \mathrm{mG} / \mathrm{V}$. This study was performed using an axially polarized PZT phase, limiting the transferred strain across the Terfenol-D cylinder interface to that from Poisson's effect. Youssef et al. studied the same structure to remedy this limitation but with a radially polarized PZT cylinder to achieve direct piezoelectric strain transfer [13]. Contrary to their original hypothesis that this higher strain would transfer to the Terfenol-D cylinder and result in a higher overall magnetoelectric coupling, the CME of the radially polarized PZT composite was limited to $282 \mathrm{mG} / \mathrm{V}$. They attributed this clipping of the output to a mechanical clamping force that altered the magnetostrictive response of the Terfenol-D cylinder within the investigated frequency and bias magnetic field ranges [13]. Newacheck et al. continued the investigations of Chavez et al. and Youssef et al. to identify the underpinnings of the hypothesized clamping force through the application of a concurrent multidirectional bias magnetic field [14]. Their study detailed the CME response based on the direction of the applied electric field and the magnetocrystalline anisotropy of the composite phases and confirmed that the clamping force limited the overall ME coupling [14]. In addition to the investigation of the bilayer cylinders, others have recently studied tricylinder structures using different assembly techniques, as delineated in $[15,16]$. Interestingly, Newacheck et al. recently discovered an extended frequency-modulated operation range of concentric multiferroic cylinders beyond the magnetic field required to achieve the peak piezomagnetic response and magnetic saturation [17]. The culmination of these studies provides the experimental validation of the standing hypothesis by Bichurin and Viehland regarding cylinder structures outperforming their 2-2 laminated plate counterparts [12-14,17-19].

Despite the recent influx of research on strain-mediated multiferroic composite structures, there is a gap in the knowledge on their long-term performance, hence the objective of the present study. Here, a predefined crack was created at the interface between the two material phases, i.e., within the epoxy layer. The composite structure was operated under the converse magnetoelectric coupling paradigm. It was subjected to a continuous load (exceeding one hundred billion cycles) under the influence of an electric field at a near-resonant frequency and a bias magnetic field corresponding to the saturation level. In addition to quantifying the emanating magnetic flux at the crack site and a diametrically opposed location, the temperature change was continually monitored throughout the experiment. After the conclusion of testing, a rigorous postmortem characterization protocol was performed using scanning electron microscopy (SEM) to explicate the effect of extended loading on the morphology of the epoxy layer of the sample. This was then compared to the virgin, undamaged structures to glean information about the performance implications of the intentionally debonded interface and the resulting fracture propagation behavior.

\subsection{Sample Preparation}

The investigated concentric multiferroic cylinder composite structure consisted of two discrete cylinders separated by a bonding interface. For the piezoelectric component, lead zirconate titanate ceramic was used (PZT 841, APC International, Mackeyville, PA, USA). The magnetostrictive cylinder was made of Terfenol-D (Etrema/TdVib, Ames, IA, USA) with a standard composition of $\mathrm{Tb}_{0.3} \mathrm{Dy}_{0.7} \mathrm{Fe}_{1.92}$ [20]. A highly conductive silver-filled 
two-part epoxy (MG Chemicals 8330S, Ontario, ON, Canada) was used to mechanically bond the two cylinders.

The as-received dimensions of the Terfenol-D cylinder were a $20.03 \mathrm{~mm}$ inner diameter (ID), a $25.02 \mathrm{~mm}$ outer diameter (OD), and a height of $10.01 \mathrm{~mm}$. Since Terfenol-D is extremely brittle, the only prebonding processing step was a perfunctory removal of the naturally occurring oxide layer using 400-grit sandpaper. The as-received, radially polarized PZT cylinder had an ID of $24.73 \mathrm{~mm}$, an OD of $30.24 \mathrm{~mm}$, and a height of $5.01 \mathrm{~mm}$. Preattached conductive silver electrodes on the interior and exterior faces were used during the manufacturing process for polarization and were left attached to facilitate an electric field during normal operation. The inner diameter of the PZT cylinder was carefully and evenly sanded to achieve a uniform, concentric fit between the two cylinders. A 25- $\mu \mathrm{m}$-thick skived Teflon (polytetrafluoroethylene (PTFE)) spacer was inserted between the two cylinders to create a predefined crack to investigate the effect that extended loading had on the fracture propagation. Subsequently, the two-part silver-filled conductive epoxy was applied liberally, with care taken to avoid air pockets or voids, and then heat-cured for two hours at $65^{\circ} \mathrm{C}$. Following curing, the Teflon spacer was carefully removed to reveal the predefined crack. Given the initial height difference between the two cylinders, the sample was wet sanded to a final composite height of $4.82 \mathrm{~mm}$. Figure 1 succinctly and schematically documents the preceding sample preparation process.
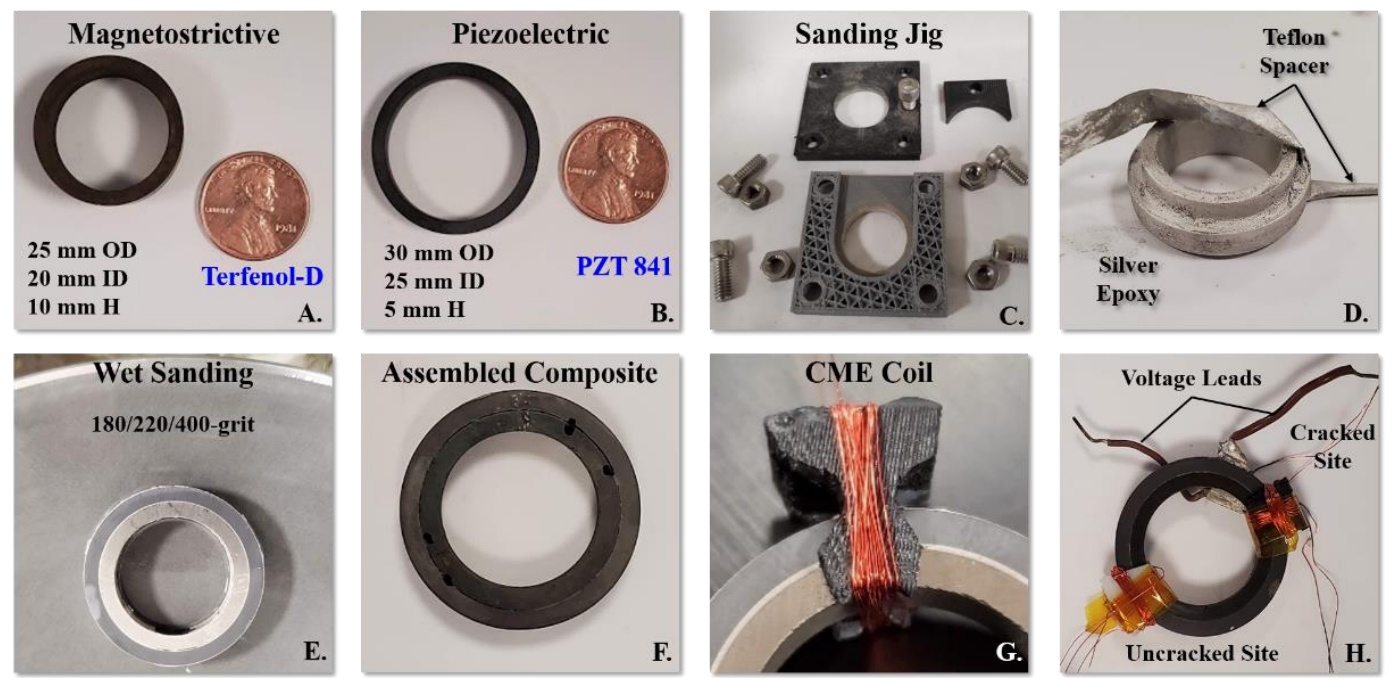

Figure 1. Overview of the sample preparation process showing (A) a Terfenol-D cylinder with the nominal dimensions, (B) a PZT cylinder with the as-received dimensions, (C) a 3D-printed sanding jig for adjusting the size of the PZT cylinder to fit onto the Terfenol-D cylinder, (D) an epoxy bonding step showing the Teflon spacer prior to removal, (E) the result of the wet-sanding process to flatten the faces, $(\mathbf{F})$ the final assembled composite cylinder, $(\mathrm{G})$ the assembled search coil to monitor the $\mathrm{CME}$, and $(\mathrm{H})$ the composite structure with voltage leads for the AC electric field and the two CME coil locations that were ready for testing.

The sample was then fitted with two search coils to measure the electromotive force (emf) at the cracked and uncracked sites, where a 36 AWG polymer-sheathed copper magnet wire was used to create the 16-turn coils. Electrodes were attached to the outer and inner diameters of the composite cylinder using the same silver epoxy that was used to bond the cylinders to apply the AC electric field.

\subsection{Micrographic Analysis}

The micrographic analysis using scanning electron microscopy (FEI Quanta 450 ESEM, Hillsboro, OR, USA) provided the ability to characterize the dimensions of the predefined crack and the change in its length after extended loading, as well as elucidating the evolution in the morphology of the epoxy interface. Acquiring a singular SEM image of 
the entire structure was not possible given the dimensions of the composite cylinder with an outer diameter of $30.24 \mathrm{~mm}$ and an inner diameter of $20.03 \mathrm{~mm}$. Therefore, 230 separate SEM micrographs at a magnification of $55 \times$ were sequentially collected to contiguously map the entire surface of the sample, where a $20 \%$ overlap between successive images was used to facilitate subsequent image reconstruction. It is essential to note that SEM was used in lieu of an optical microscope since a preliminary investigation with the latter was futile. The FIJI release of ImageJ2, which is image processing software, and the TrakEM2 plugin were used to digitally stitch the images to reconstruct the geometry of the entire composite cylinder structure [21-23]. TrakEM2 is a specialized plugin that was designed to combine and analyze sets of electron microscopy images, as was the case here [22-26].

The collected images were approximately aligned to a square grid according to the $20 \%$ overlap, as shown in Figure $2 \mathrm{a}-\mathrm{c}$. The images were then montaged using the least-square linear feature correspondence method and translation alignment [26]. The SIFT (scaleinvariant feature transform) parameters and geometric consensus filtering parameters, which are responsible for feature detection and image alignment, respectively, that were used herein are shown in Figure 2d,e [23,26]. The feature descriptor size and maximal alignment error are key parameters for the overall quality of the final image due to the relative lack of distinctive features between images of the composite, and the initial grid alignment process described previously. Once all tiles were aligned, the relative intensities were unified, a blending process was used to combine the tiles into a single image without exceedingly pronounced changes in the per-tile brightness and contrast.
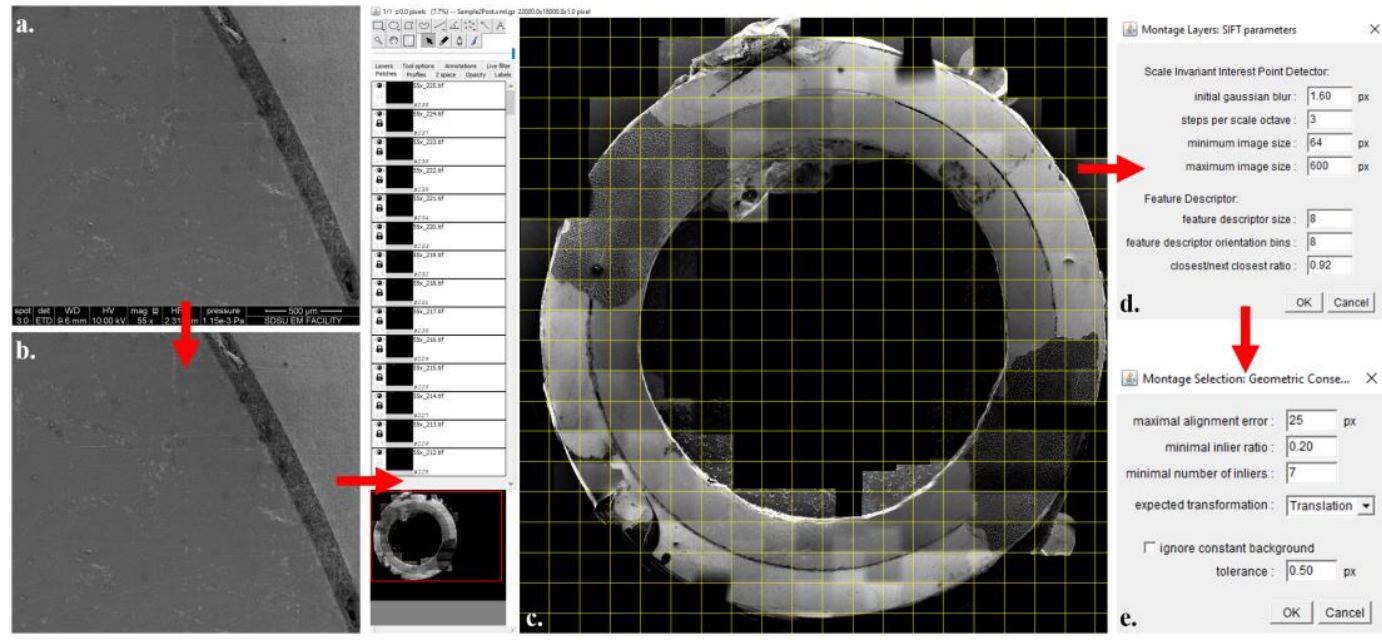

Figure 2. SEM image reconstruction process using ImageJ2/TrakEM2, where (a) an original SEM micrograph was (b) cropped with removed labels, (c) the approximate alignment of the image was set using a square grid, (d) SIFT parameters were used for feature detection, and (e) geometric consensus filter parameters were used to control the tile alignment.

As a result of the reconstruction step, the stitched SEM images of the entire multiferroic cylinder structure were created before and after testing, showing the overall dimensional changes of the composite cylinder with a focus on the interface. It is worth noting that despite the concerted and careful efforts in the alignment process, the reconstructed images have two major artifacts, namely, missing sections due to the movement of the electron beam and misalignment between successive images and contrast discrepancies between image tiles due to the different interactions between the electron beam and the three materials (PZT, Terfenol-D, and silver epoxy). These artifacts were deemed cosmetic with no effect on the subsequent analyses; the contrast artifacts did not affect the results reported herein, given the distinct interaction between each of the materials and the electron beam. 


\subsection{CME Measurement Setup}

The sample was continuously loaded, electrically and magnetically, over 45 days with a DC magnetic field of 500 Oe and a simultaneous AC electric field of $20 \mathrm{kV} / \mathrm{m}$ at a frequency of $32.5 \mathrm{kHz}$ to study the long-term performance of the multiferroic cylinder composite structure. These conditions were selected based on a priori experimental studies [12-14,17-20,27-29]. The sample was held in place using a 3D-printed mount that was centered perpendicularly between the two poles of an electromagnet. The sample mount was designed to provide the minimum amount of mechanical constraint possible by loosely supporting the cylinder composite from its inside diameter while being magnetically transparent, negating any effects on the magnetic response [14]. The composite cylinder was oriented such that the bias magnetic field was diametrically applied using an electromagnet (GMW Associates 3470, San Carlos, CA, USA) and a Sorensen DLM 6010 power supply (San Diego, CA, USA), whereas the search coils wrapped around the cracked and uncracked sites were located at the zenith and nadir locations (Figure $3 b$ ). It has been experimentally and computationally shown that multiferroic cylinder composites experience the largest strains in these regions $[17,19,30]$. The bias magnetic field was monitored using a bias field probe (FW Bell HTR81-0608-10, Portland, OR, USA) connected to a gaussmeter (FW Bell 3030, Portland, OR, USA). The field probe tip was placed near the leading edge of the sample to ensure that the bias field that was measured was representative of the bias field for the entire sample. The AC electric field was applied using a high-voltage amplifier (Advanced Energy Trek 609B-3, Denver, CO, USA) that was connected to a function generator (Hewlett-Packard 33210A, Palo Alto, CA, USA). The magnitude and frequency of the AC electric field were verified using a high-voltage oscilloscope (Tektronix DPO-2012B, Beaverton, OR, USA).

a.

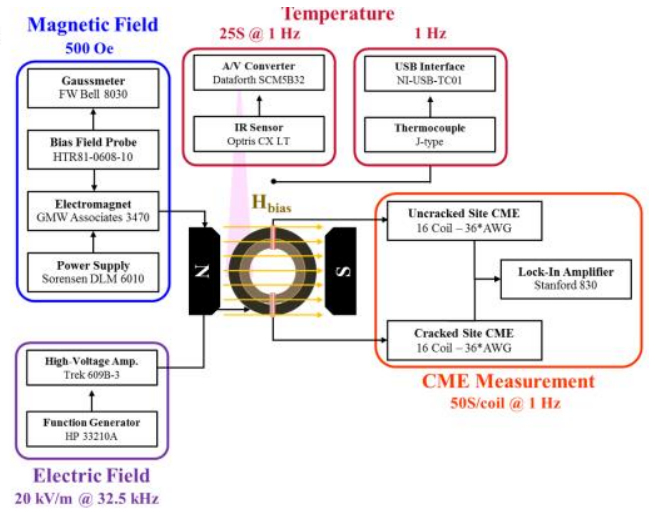

b.

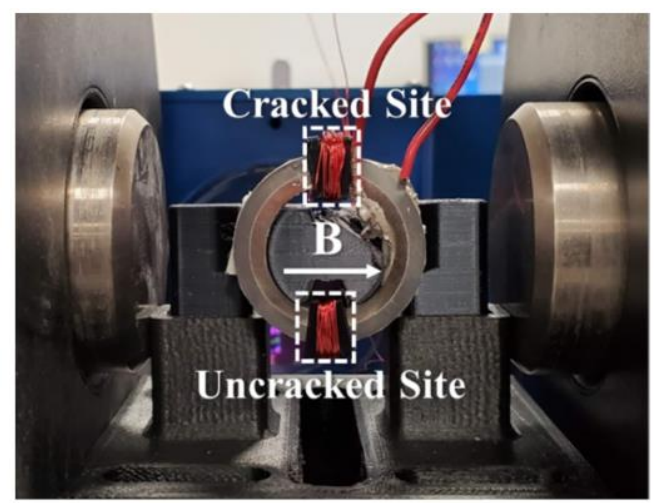

Figure 3. (a) Schematic diagram overview of the experimental setup including loading and sampling parameters, and (b) multiferroic composite cylinder in the testing configuration with electromagnet poles, a coil mount, and CME coils visible.

The signals from the search coils (used to calculate the CME) were collected using a lock-in amplifier (Stanford Research Systems 830, Sunnyvale, CA, USA) and a synchronized trigger signal from the function generator (Hewlett-Packard 33210A). Readings from both coils were averaged 25 times per second for a final sample rate of $1 \mathrm{~Hz}$. Finally, temperature measurements were made using a fixed-focus IR sensor (Optris PSC-CX LT, Portsmouth, $\mathrm{NH}, \mathrm{USA}$ ) pointed near the crack region to monitor localized temperature changes. The IR sensor data was sampled 50 times per second, with the readings averaged to output a $1 \mathrm{~Hz}$ data rate.

\section{Results and Discussion}

The results section is divided into two subsections corresponding to the two main forms of analyses discussed above, starting with the micrographic analysis based on the SEM imaging, followed by a discussion of the CME response of the composite structure over 
the extended loading period. Given the frequency of the applied electric field $(32.5 \mathrm{kHz})$ and the total testing duration (45 days), in total, the multiferroic concentric cylinder structure was subjected to 126 billion fully reversed loading cycles. The relatively high number of cycles is consistent with the lifetime of electronic devices.

\subsection{Results of the Micrographic Analysis}

Figure 4 shows the stitched SEM images of the sample before loading (Figure $4 \mathrm{a}$ based on 230 SEM micrographs) and after the extended loading period (Figure $4 \mathrm{~b}$ based on $226 \mathrm{SEM}$ micrographs) as a result of the reconstruction steps discussed in the previous section.

a.



b.

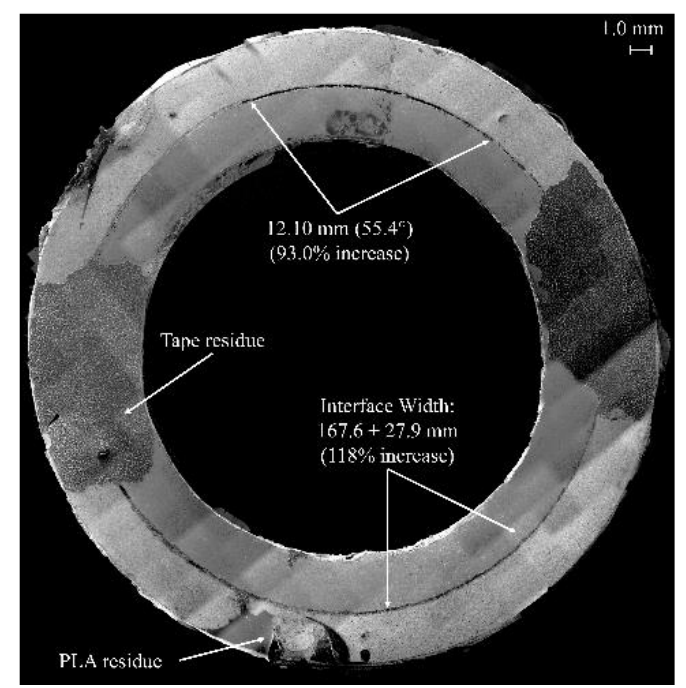

Figure 4. Stitched SEM images of the multiferroic cylinder composite (a) before testing, where the region of the predefined crack is annotated (here, black squares are areas of missing image data), and (b) after the 45-day period of cyclic loading. Degradation of the epoxy interface quality is clearly visible, as evidenced by the widespread void formation and initial crack lengthening.

The arc length of the predefined crack before loading was found to be $6.27 \mathrm{~mm}$ (Figure 5a), corresponding to a circumferential angle of $28.7^{\circ}$ and representing a $2.27 \mathrm{~mm}$ $\left(10.5^{\circ}\right)$ increase of the planned length of $4.0 \mathrm{~mm}$. The difference between the realized and planned crack lengths was attributed to the possible shifting of the Teflon spacer during the manufacturing process, given that the silver epoxy was very malleable before curing. To further characterize the crack, the crack tips were measured and found to have respective thicknesses of 43 and $47 \mu \mathrm{m}$, indicating that the two crack tips shared similar characteristic sizes. The last notable geometrical attribute was the thickness of the silver epoxy bonding interface, which was of paramount importance as it was the basis of the strain mediation and resultant magnetoelectric response of the composite structure. Figure $5 \mathrm{c}$ shows a representative interface between the composite phases, which was consistent with all other SEM micrographs collected around the circumferential interface. Common to the entire circumference (except where the crack was introduced) was the excellent interface quality, where no significant voids, defects, or pre-existing bond separation were observed. The average interface width of $76.8 \pm 12.8 \mu \mathrm{m}$ was based on collected measurements at $20^{\circ}$ increments around the circumference. The interface width difference was attributed to (1) the hand-sanding process that was used to fit the two rings and (2) the malleability of the silver epoxy that was used to assemble the cylinders. 

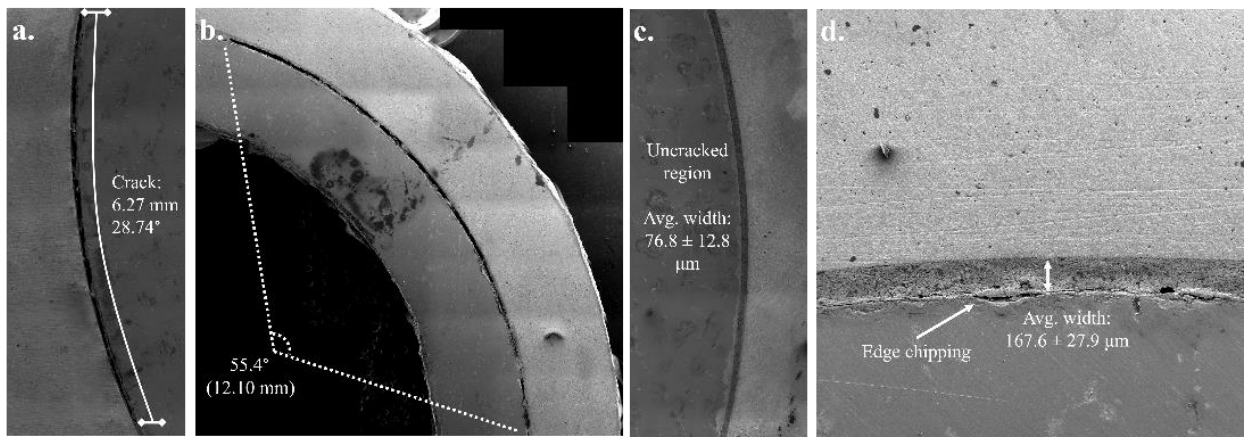

Figure 5. A collection of SEM micrographs of selected areas before and after the testing showing (a) the arc length of the predefined crack before testing of $6.27 \mathrm{~mm}$, which (b) extended to $12.10 \mathrm{~mm}$ after 45 days of continuous loading, (c) a close-up view of the bonding layer showing the initial thickness of $76.8 \pm 12.8 \mu \mathrm{m}$, and (d) the thickness of the bonding layer that had increased to $167.6 \pm 27.9 \mu \mathrm{m}$ after the testing.

Upon testing and removing the samples from the experimental setup, the electrode wires and search coils were carefully detached from the composite structure. Without further processing, the sample was reexamined using the electron microscope, closely following the steps used in characterizing the virgin samples. With a focus on the interfaces and the bonding layer, the post-stitching analysis revealed three overarching observations:

1. The arc length of the predefined crack, which was the area of the most significant interface debonding at the location of the Teflon strip, increased to $12.10 \mathrm{~mm}$ from an initial length of $6.27 \mathrm{~mm}$ (Figure 5a), i.e., an increase of $93.0 \%$ from the initial crack length, corresponding to a total angle of $55.4^{\circ}$. The crack length nearly doubled due to the oscillatory nature of the loading, where the applied AC electric field resulted in cyclic strains that continuously loaded the samples and induced corresponding oscillatory magnetostriction in the inner Terfenol-D cylinder. In other words, the mechanical work provided by the generated piezoelectric strain and the induced piezomagnetic strain cyclically loaded the predefined crack to failure.

2. Gross debonding was noticeable along the interfaces, particularly at the silver epoxy and Terfenol-D boundary. It is worthwhile to note that close examination of the tested sample confirmed that the debonding sites were a byproduct of the long-term testing.

3. The quality of the bonding layer at the onset of testing was noted to be 'excellent' as the interface was free of voids or cracks, except for the predefined crack. However, the bonding layer quality noticeably degraded during testing, as evidenced in Figure 6 by the nucleation of voids and cracks within the bonding layer, which was attributed to the epoxy cohesion failure.

a.

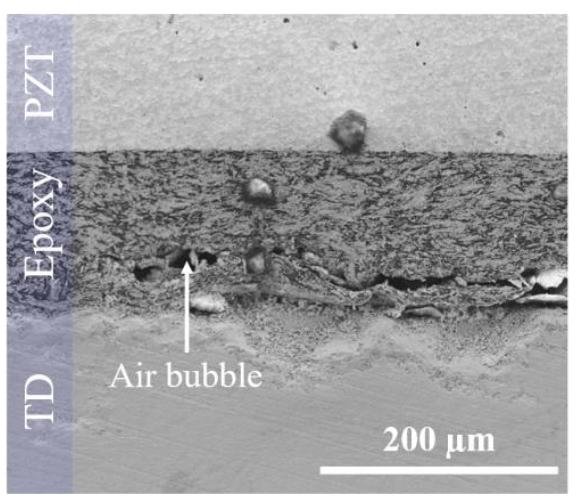

b.

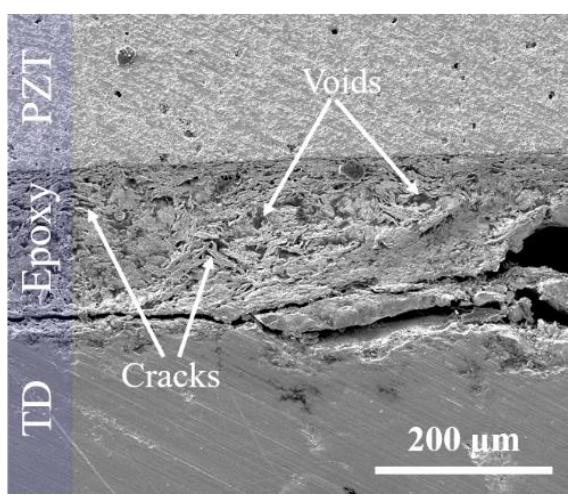

Figure 6. SEM micrograph of the epoxy interface sandwiched between the magnetostrictive TerfenolD and piezoelectric PZT layers (a) before electrically and magnetically loading the sample and (b) after the 45-day period of cyclic loading. 
Additionally, a high magnification surface inspection of the Terfenol-D and PZT cylinders showed that the cracks (new or predefined) were bounded within the silver epoxy bonding layer, where the active cylinders maintained the same morphology, except for a few chipping sites at the outer rim of the Terfenol-D ring. Overall, the predefined crack did not propagate through the bonding layer. Instead, it evolved to an interfacial crack that was exhibited in over $90 \%$ of the circumferential interface, as shown in Figure $4 \mathrm{~b}$.

Based on the SEM micrographs measurements leading to Figures 4 and 5, there was a striking change in the thickness of the bonding layer separating the two cylinders throughout testing. The as-fabricated thickness of the silver epoxy layer was $76.8 \pm 12.8 \mu \mathrm{m}$, while the thickness of the same layer after dynamic testing was found to be $167.6 \pm 27.9 \mu \mathrm{m}$, constituting a $118 \%$ increase in the bonding layer thickness. This difference resulted from changes in the Terfenol-D ring dimension, which was measured to have an average outer diameter of $24.82 \mathrm{~mm}$ after testing instead of $24.99 \mathrm{~mm}$ before testing. As discussed in the experimental protocol, the testing stimuli included an AC electric field that was applied radially to generate piezoelectric strain, which was transferred across the interface to induce piezomagnetic strain in the inner Terfenol-D ring. Additionally, the latter was subjected to a diametrically applied bias magnetic field of $500 \mathrm{Oe}$, corresponding to the onset of magnetic saturation of the material [19]. In summary, the Terfenol-D ring was under the combined influence of compressive stress and bias magnetic field for an extended duration and, as a result, permanently and nearly uniformly radially contracted.

In an effort to mechanistically explain the predominance of the interface debonding at the silver epoxy/Terfenol-D interface with the lack of kinking, the framework of Dundurs' parameters from linear elastic fracture mechanics of multilayered structures was brought into consideration. Dundurs' parameters describe the strain distributions and, therefore, crack-branching behavior in a discrete composite as a function of the relative properties of each material phase [31-33]:

$$
\alpha=\frac{\bar{\mu}\left(\kappa_{1}+1\right)-\left(\kappa_{1}+1\right)}{\bar{\mu}\left(\kappa_{1}+1\right)+\left(\kappa_{1}+1\right)} \text { and } \beta=\frac{1}{2} \frac{\mu_{1}\left(1-2 v_{2}\right)-\mu_{2}\left(1-2 v_{1}\right)}{\mu_{1}\left(1-2 v_{2}\right)+\mu_{2}\left(1-2 v_{1}\right)}
$$

where $\bar{\mu}=\frac{\mu_{2}}{\mu_{1}}, \kappa_{i}=\frac{3-v_{i}}{1+v_{i}}, \mu$ is the shear modulus (taken to be 19 GPa for PZT, $0.3 \mathrm{GPa}$ for silver epoxy, and $12 \mathrm{GPa}$ for Terfenol-D), and $v_{\mathrm{i}}$ is Poisson's ratio (taken to be 0.32 for PZT, 0.40 for silver epoxy, and 0.25 for Terfenol-D). Regardless of the investigated interface, the parameters $\alpha$ and $\beta$ from Equation (1) were found to be 0.96 and 0.16, respectively. Since $\alpha$ is close to unity for both interfaces, this suggested that interface debonding was the most probable form of failure, which was consistent with the results explicated in the previous section. Composite interfaces with $\alpha(\alpha-2 \beta)>0$ are characterized by compression of the interface layer due to an elastic mismatch. In this configuration, as was the case for both interfaces under consideration herein, an increase in the shear stresses was predicted, leading to the increased likelihood of interfacial crack formation [31-33]. Since $\beta$ was nonzero, this suggested minor crack kinking in the crack tips, which was observed in some locations around the outer diameter of the Terfenol-D cylinder.

The prominence of the interfacial cracks at the silver epoxy/Terfenol-D interface was attributed to the contraction of the outer diameter of the Terfenol-D ring, which also resulted in the cohesion failure that was sporadically exhibited within the bonding layer. As the outer diameter contracted, it pulled the bonding layer in the radial direction, hence the highlighted failure modes. While the Dundurs' parameters indicated a near-equal opportunity for either interface failure, the failure mode dominance along the Terfenol-D is explained by the preceding discussion. Furthermore, sanding of the inner diameter of the PZT ring resulted in better bonding at the PZT/silver epoxy interface. The latter implies that mechanical interlocking at the PZT/silver epoxy interface played a major role in improving the interfacial strength at that interface and resulted in a low probability of debonding, as discussed before. On the other hand, the bonding surface of the Terfenol-D ring, i.e., the outer diameter of the ring, was only gently buffed to remove surface oxidation and avoid breakage of the intrinsically brittle material. 


\subsection{The CME Response}

Figure 7 shows the converse magnetoelastic coupling coefficient of the composite cylinder structure, which was monitored at the crack site and a diametrically opposed location. The CME was reported over 45 days of continuous actuation of the external PZT cylinder and under the influence of a bias magnetic field. The axis of the search coils fitted around the cylinder coincided with the direction of the bias magnetic field. By holistically considering the CME shown in Figure 7, it is worth noting the following three overarching observations. First, the average CME over the entire actuation period at the uncracked site was around $75 \%$ higher than at the cracked location, whereas the CME at the uncracked site was $342.5 \pm 90.1 \mathrm{mG} / \mathrm{V}$, while it was $193.3 \pm 29.9 \mathrm{mG} / \mathrm{V}$ at the cracked site. The former figure was found to be initially higher than the CME reported by Chavez et al. for a similar fully intact composite structure in the first week of testing, but on average, it is in good agreement [12]. The difference in the results reported herein compared to [12] was attributed to the enhanced sample preparation process, as discussed in the previous section. The disparity between the CME values at the observation sites was attributed to the presence of the predefined crack, given that the main mediator between electric and magnetic energies in the investigated multiferroic paradigm is the mechanical strain. In other words, the quality of the bonding layer was compromised at the crack site, which reduced the efficacy of strain transduction and resulted in a lower CME. The poor strain transfer at the crack site was consistent with the predictions of the effective medium theory and previous experimental investigations $[5,12,13,17-19,27,34,35]$. A final note about the first observed behavior of the CME is warranted since it is counterintuitive to expect any strain-mediated coupling whatsoever at the crack site given the reasons regarding interface quality and the dependence of transduction efficacy of the continuity conditions, as discussed above. The ability to measure and report magnetoelectric coupling at the crack site is associated with the self-boundedness of the cylinder geometry rather than its sole reliance on interface quality, as is the case in other composite configurations, namely 2-2 laminated plates. Here, the self-boundedness effect was defined as the high efficiency of strain mediation between the ferromagnetic and piezoelectric materials because of the geometry and the directionality of strain transfer in the composite. That is, the circumferential in-plane and out-of-plane vibrational modes were coupled and continuous within the continuum of the cylinder, as was recently shown experimentally and numerically in [17], which gave rise to a local magnetostrictive strain in the proximity of the crack, hence, coupling electric and magnetic energies.

Second, from the onset of testing until nearly 3 min of loading, the CME at both observation locations was found to increase nearly monotonically to reach $223.2 \mathrm{mG} / \mathrm{V}$ and $432.5 \mathrm{mG} / \mathrm{V}$ for the cracked and uncracked locations, respectively. While the CME at the crack site increased at a rate of $183 \mu \mathrm{G} / \mathrm{V} \cdot \mathrm{s}$, reaching the maximum after $160 \mathrm{~s}$, the CME at the uncracked site ascended at a rate of $472 \mu \mathrm{G} / \mathrm{V} \cdot \mathrm{s}$ to the peak value after $175 \mathrm{~s}$. The quasi-linear increase of the $\mathrm{CME}$, regardless of the location or rate, was attributed to the time required to fully develop the displacement field and associated vibrational modes in the structure after the spontaneous generation of radial piezoelectric strain upon the application of an electric field. In other words, and contrary to the supposition that is usually employed in analytical models, each constituent material had a source of internal losses, including electrical damping of the outer piezoelectric cylinder and mechanical losses in the silver epoxy bonding layer, to name a few sources of structural damping. Moreover, as additional high-frequency structural vibrations were stimulated due to the coupling of lower frequency modes, the resulting overall mechanical strain increased, thus, registering increasing values of magnetoelectric coupling until the steady-state response was reached at $160 \mathrm{~s}$ and $175 \mathrm{~s}$ for the cracked and uncracked sites, respectively. The difference in the rate of increase within this initial period was solely associated with the quality of the interface, where the uncracked sites exhibited direct, bidirectional transfer of piezoelectric and piezomagnetic strains. On the other hand, the crack site strains were transduced ahead of the crack tips at fully intact locations, then propagated through 
the magnetostriction cylinder, resulting in a localized oscillatory piezomagnetic strain under the search coil surrounding the crack site. The indirect transfer of strain around the crack site resulted in the slight observed time lag of $15 \mathrm{~s}$ between the uncracked and cracked locations.

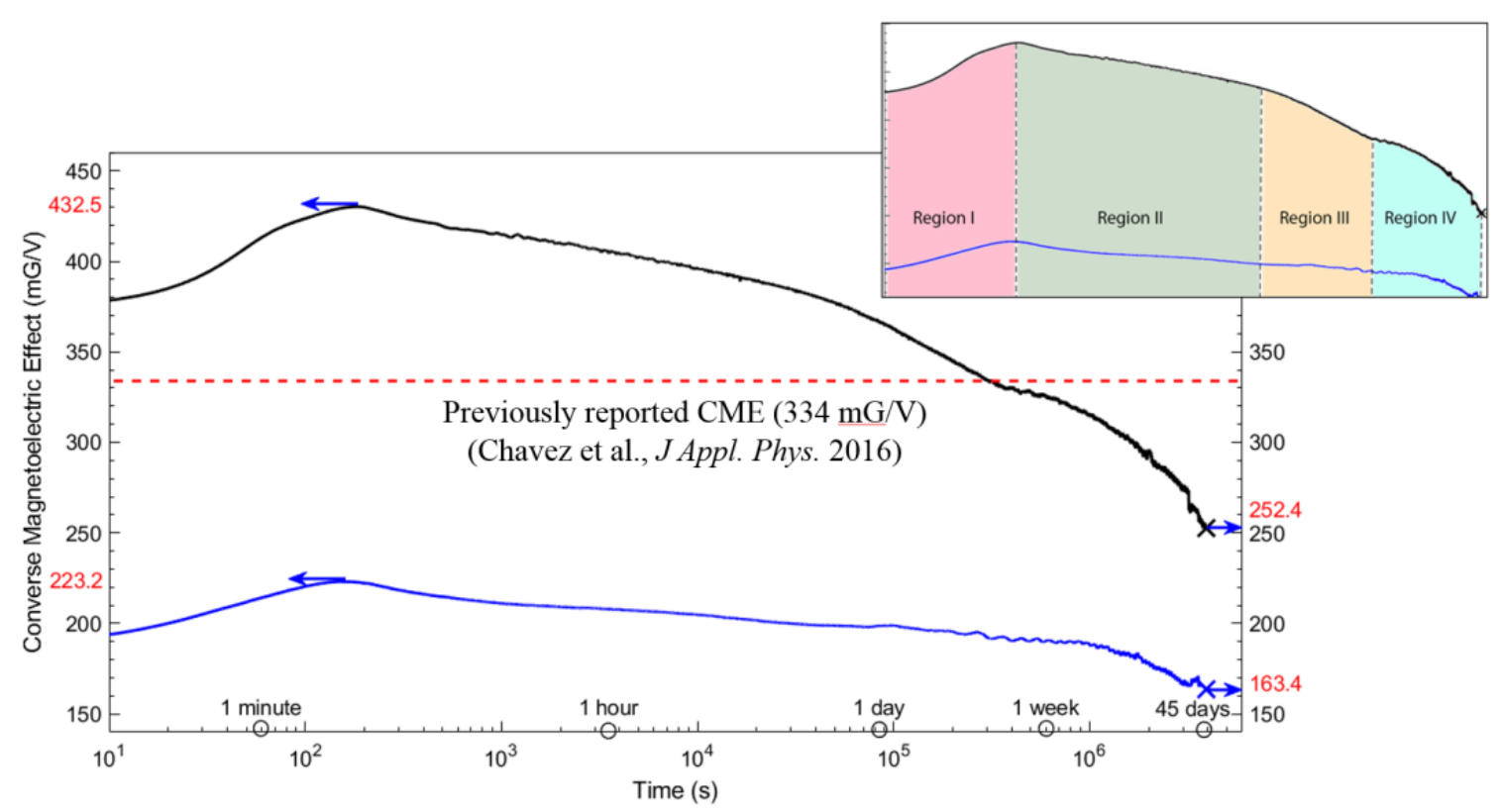

Figure 7. CME response for cracked (blue) and uncracked (black) sites and the previously reported CME of identical cylinder structure under short-term loading ([12]) is shown as a red dashed line. A time lag was observed between the initial response peaks of cracked and uncracked sites. The inset shows the regions of the magnetoelectric response.

Finally, once the CME reached its maximum values, the remainder of the response can be divided into three successive regions, where regions II and III showed a quasilinear reduction in the magnetoelectric coupling but at two different rates. Region II extended for $11 \mathrm{~h}$, over which, the CME reduced at a rate of $10.17 \mathrm{mG} / \mathrm{V}$ and $20.66 \mathrm{mG} / \mathrm{V}$ per logarithmic decade of time in seconds for the cracked and uncracked sites, respectively. On the other hand, region III was taken to last for 3.6 days preceding region IV, within which, the reduction rate of the CME values accordingly slowed down to a rate of $6.91 \mathrm{mG} / \mathrm{V}$ per decade for the cracked site, while it accelerated to nearly double at a rate of $51.66 \mathrm{mG} / \mathrm{V}$ per decade at the uncracked site. The terminal stage (region IV in Figure 7) was characterized by a rapid drop in the CME values as the testing time increased. Another characteristic of region IV is the appearance of superimposed oscillation in the response. After 45 days of testing, i.e., at the end of the testing period, the uncracked CME was found to be $255 \mathrm{mG} / \mathrm{V}$ instead of $163 \mathrm{mG} / \mathrm{V}$ for the CME at the crack site, which was a difference of only $56 \%$. This corresponded to an overall rate of decrease of $3.94 \mathrm{mG} / \mathrm{V}$ per day and $1.33 \mathrm{mG} / \mathrm{V}$ per day for the uncracked and cracked sites, respectively. The continuous reduction in the CME values at either observation site, regardless of the rate, was attributed to the degradation of the interface, where the interfacial cracks nucleated and propagated as elucidated based on the micrographic analyses. As the degradation rate of the interface quality accelerated, corresponding to region IV of Figure 7, the interfacial cracks propagated and released stress waves that continued to circulate the cylinder due to the self-boundedness effect. In other words, the incremental interfacial debonding was the source of the superimposed oscillations observed in the CME response in region IV at the cracked and uncracked locations. Table 1 succinctly summarizes the underlying characteristics of the respective regions. Future studies will extend the loading period until a catastrophic failure occurs. 
Table 1. Changes in magnetoelectric response for the response regions that are denoted in Figure 7 as a function of logarithmic time at the uncracked (UC) and cracked (C) sites.

\begin{tabular}{|c|c|c|c|c|}
\hline Region & $\mathbf{I}$ & II & III & IV \\
\hline Duration & $\begin{array}{c}175 \mathrm{~s}(\mathrm{UC}) \\
160 \mathrm{~s}(\mathrm{C})\end{array}$ & $11 \mathrm{~h}$ & 3.6 days & 41 days \\
\hline Uncracked CME (mG/V per decade) & 61.36 & -21.66 & -51.66 & -74.62 \\
\hline Cracked CME (mG/V per decade) & 23.73 & -10.17 & -6.91 & -27.45 \\
\hline
\end{tabular}

As part of the experimental protocol, the surface temperature of the composite ring structure was continuously monitored using an IR sensor that was aimed near the cracked site. At the onset of testing, the temperature of the composite structure was recorded to be $21.0{ }^{\circ} \mathrm{C}$, which reached a steady-state average of $94.3 \pm 0.4{ }^{\circ} \mathrm{C}$ over nearly the entire loading period. The increase in temperature amounted to thermal energy $(\mathrm{Q})$ of $\approx 216 \mathrm{~J}$ due to the specific heat capacity (c, taken to be $350 \mathrm{~J} / \mathrm{kg} \cdot \mathrm{K}$ ) of the piezoelectric material $(\mathrm{Q}=m c \Delta T)$. The increase in temperature was associated with energy losses (discussed above due to damping) and was accounted for by using the strain energy density (U) due to the piezoelectric strain, such that:

$$
U=\frac{\pi}{2} Y d^{2}\left(\frac{V}{\delta}\right)^{2}
$$

where, $Y, V, d$, and $\delta$ are the elastic modulus of PZT (76 GPa), the amplitude of the applied voltage $(100 \mathrm{~V})$, the piezoelectric coefficient $\left(3 \times 10^{-10} \mathrm{~m} / \mathrm{V}\right)$, and the wall thickness of the sample in the polarization direction $(2.5 \mathrm{~mm})$, respectively. The total strain energy release was found to be $\approx 782$ J over the first $\approx 2600 \mathrm{~s}$ of actuation. Overall, the thermal energy from the increase in temperature constituted nearly $28 \%$ of the strain energy over the same period, which was found to be in excellent agreement with the previous results of Zheng et al., which reported a 30\% energy loss in piezoelectric materials due to Joule heating [36].

In closing, several follow-up studies are planned to explicate the effect of the initial crack on the fracture behavior as a function of the loading times. As part of such an effort, the length of the initial crack can be controlled by adjusting the width of the Teflon spacer used when introducing the crack (see Section 1.1 above). Another emphasis of future studies is elucidating the electric field effect, which can be used to tune the induced strain (i.e., the input mechanical energy). Increasing the electric field may result in different fracture behavior than that reported above, hence the motivation for future investigations.

\section{Conclusions}

In conclusion, the research performed in this study was designed to resolve a fundamental gap in the understanding of the long-term performance of multiferroic composite structures since devices based on this coupling paradigm are expected to undergo billions of cycles. The most remarkable outcome of the study was the elucidation of a measurable converse magnetoelectric coupling coefficient at a location of a predefined crack in the bonding layer, which was primarily attributed to the self-boundedness effect of the cylinder structure. Furthermore, this study demonstrated that these structures experienced significant degradation in the magnetoelectric response under extended loading. Over the 45 -day testing period, the CME decreased by $48.4 \%$ at the uncracked site but only $26.7 \%$ at the cracked site. The micrographic analysis additionally confirmed the interface bonding and void nucleation fatigue-related behaviors and suggested the existence of additional aging effects that deserve further investigation. While the experimental studies in this research investigated samples for up to 45 days, corresponding to 126 billion loading cycles, there were still ongoing changes in the magnetoelectric response observed at the end of 
this period. Future research studies will extend the loading period until catastrophic failure is observed to elucidate the device lifetime reliability.

Author Contributions: Conceptualization, G.Y. and R.S.; methodology, R.S. and N.U.H.; software, R.S.; validation, G.Y. and R.S.; formal analysis, G.Y., R.S. and N.U.H.; investigation, R.S. and N.U.H.; resources, G.Y.; data curation, R.S. and N.U.H.; writing-original draft preparation, G.Y. and R.S.; writing-review and editing, R.S. and N.U.H.; visualization, G.Y. and R.S.; supervision, G.Y.; project administration, G.Y. and R.S.; funding acquisition, G.Y. All authors have read and agreed to the published version of the manuscript.

Funding: This research received no external funding.

Data Availability Statement: The data presented in this study are available on request from the corresponding author. The data are not publicly available due to privacy.

Acknowledgments: The authors are grateful to the SDSU Electron Microscope Facility, with data acquired under the support of a National Science Foundation grant (DBI-0955908). The authors also acknowledge the insightful discussions with Scott Newacheck of the Experimental Mechanics Laboratory.

Conflicts of Interest: The authors declare no conflict of interest.

\section{References}

1. Ramesh, R.; Spaldin, N.A. Multiferroics: Progress and prospects in thin films. In Nanoscience and Technology: A Collection of Reviews from Nature Journals; World Scientific: Singapore, 2009; ISBN 9789814287005.

2. Spaldin, N.A.; Fiebig, M. The renaissance of magnetoelectric multiferroics. Science 2005, 309, 391-392. [CrossRef] [PubMed]

3. Spaldin, N.A.; Ramesh, R. Advances in magnetoelectric multiferroics. Nat. Mater. 2019, 18, 203-212. [CrossRef]

4. Ramesh, R.; Spaldin, N.A. Multiferroics: Progress and prospects in thin films. Nat. Mater. 2007, 6, 20-29. [CrossRef]

5. Nan, C.W.; Bichurin, M.I.; Dong, S.; Viehland, D.; Srinivasan, G. Multiferroic magnetoelectric composites: Historical perspective, status, and future directions. J. Appl. Phys. 2008, 103, 1. [CrossRef]

6. Vopson, M.M. Fundamentals of Multiferroic Materials and Their Possible Applications. Crit. Rev. Solid State Mater. Sci. 2015, 40, 223-250. [CrossRef]

7. Palneedi, H.; Annapureddy, V.; Priya, S.; Ryu, J. Status and perspectives of multiferroic magnetoelectric composite materials and applications. Actuators 2016, 5, 9. [CrossRef]

8. Laughlin, D.E.; Willard, M.A.; McHenry, M.E. Magnetic Ordering: Some Structural Aspects. Phase Transform. Evol. Mater. 2000, 121-137.

9. Wang, Y.; Hu, J.; Lin, Y.; Nan, C.W. Multiferroic magnetoelectric composite nanostructures. NPG Asia Mater. 2010, 2, 61-68. [CrossRef]

10. Hu, J.; Domann, J.P.; Liang, C.; Keller, S.; Carman, G.P. Sepulveda, Modeling Incoherent Strain Mediated Multiferroic Bennett Clocking. arXiv 2020, arXiv:2001.01405.

11. Tressler, J.F.; Alkoy, S.; Newnham, R.E. Piezoelectric sensors and sensor materials. J. Electroceramics 1998, 2, 257-272. [CrossRef]

12. Chavez, A.C.; Lopez, M.; Youssef, G. Converse magneto-electric coefficient of concentric multiferroic composite ring. J. Appl. Phys. 2016, 119, 233905. [CrossRef]

13. Youssef, G.; Lopez, M.; Newacheck, S. On the effect of polarization direction on the converse magnetoelectric response of multiferroic composite rings. Smart Mater. Struct. 2017, 26, 037003. [CrossRef]

14. Newacheck, S.; Webster, T.; Youssef, G. The effect of multidirectional bias magnetic fields on the converse magnetoelectric response of multiferroic concentric composite ring. Appl. Phys. Lett. 2018, 113, 172902. [CrossRef]

15. Ge, X.H.; Ji, H.; Li, Y.; Chen, J.K.; Wang, Y.G. Diameter and sequence effects on magnetoelectric effect in $\mathrm{FeCo} / \mathrm{Pb}(\mathrm{Zr}, \mathrm{Ti}) \mathrm{O}_{3} / \mathrm{Ni}$ trilayered long cylindrical composite structures. J. Alloy. Compd. 2018, 752, 303-307. [CrossRef]

16. Shen, H.Q.; Wang, Y.G.; Xie, D.; Cheng, J.H. Magnetoelectric effect in FeCo/PMN-PT/FeCo trilayers prepared by electroless deposition of FeCo on PMN-PT crystals with various orientations. J. Alloy. Compd. 2014, 610, 11-14. [CrossRef]

17. Newacheck, S.; Youssef, G. Noncontact Spatiotemporal Strain Mapping of Composite Multiferroic Cylinders. Exp. Mech. 2020, 16, 857-868. [CrossRef]

18. Newacheck, S.; Youssef, G. Wireless energy transfer based on strain-mediated composite multiferroics. Smart Mater. Struct. 2019, 29, 015014. [CrossRef]

19. Youssef, G.; Newacheck, S.; Lopez, M. Mapping magnetoelastic response of terfenol-D ring structure. Appl. Phys. Lett. 2017, 110, 1-6. [CrossRef]

20. TdVib LLC (Etrema) Terfenol-D. Available online: http:/ /tdvib.com/terfenol-d/ (accessed on 20 May 2020).

21. Rueden, C.T.; Schindelin, J.; Hiner, M.C.; DeZonia, B.E.; Walter, A.E.; Arena, E.T.; Eliceiri, K.W. ImageJ2: ImageJ for the next generation of scientific image data. BMC Bioinform. 2017, 18, 1-26. [CrossRef] [PubMed] 
22. Schindelin, J.; Arganda-Carreras, I.; Frise, E.; Kaynig, V.; Longair, M.; Pietzsch, T.; Preibisch, S.; Rueden, C.; Saalfeld, S.; Schmid, B.; et al. Fiji: An open-source platform for biological-image analysis. Nat. Methods 2012, 9, 676-682. [CrossRef] [PubMed]

23. Cardona, A.; Saalfeld, S.; Schindelin, J.; Arganda-Carreras, I.; Preibisch, S.; Longair, M.; Tomancak, P.; Hartenstein, V.; Douglas, R.J. TrakEM2 software for neural circuit reconstruction. PLoS ONE 2012, 7, e38011. [CrossRef]

24. Saalfeld, S.; Fetter, R.; Cardona, A.; Tomancak, P. Elastic volume reconstruction from series of ultra-thin microscopy sections. Nat. Methods 2012, 9, 717-720. [CrossRef]

25. Saalfeld, S.; Cardona, A.; Hartenstein, V.; Tomančák, P. As-rigid-as-possible mosaicking and serial section registration of large ssTEM datasets. Bioinformatics 2010, 26, i57-i63. [CrossRef] [PubMed]

26. Cardona, A.; Douglas, R.J.; Preibisch, S.; Saalfeld, S. TrakEM2 User Manual. Available online: https://www.ini.uzh.ch/ $\sim\{\}$ acardona/trakem2_manual.html (accessed on 20 May 2020).

27. Lopez, M.; Youssef, G. Converse magneto-electric coefficient of composite multiferroic rings. In Mechanics of Composite and Multi-Functional Materials; Springer International Publishing: New York, NY, USA, 2017; Volume 7, pp. 185-191.

28. Youssef, G.; Somer, N.; Newacheck, S. Dynamic Magnetoelectric Response of Composite Multiferroics Cylinders. Smart Mater. Struct. 2020, 29, 035025. [CrossRef]

29. Clark, A.E.; Teter, J.P.; Wun-Fogle, M. Characterization of Terfenol-D for magnetostrictive transducers. J. Acoust. Soc. Am. 1991, 89, 1448-1455. [CrossRef]

30. Stampfli, R.; Youssef, G. Multiphysics Computational Analysis of Multiferroic Composite Ring Structures. Int. J. Mech. Sci. 2020, 17, 105573. [CrossRef]

31. Dundurs, J. Edge-bonded dissimilar orthogonal elastic wedges under normal and shear loading. J. Appl. Mech. Trans. ASME 1964, 35, 460-466. [CrossRef]

32. Schmauder, S.; Meyer, M. Correlation between Dundurs' parameters and elastic constants. Z. Fuer Met. Res. Adv. Tech. 1992, 83, 524-527.

33. Shaik, A.M.; Huynh, N.U.; Youssef, G. Micromechanical behavior of ultraviolet-exposed polyurea. Mech. Mater. 2020, 140, 103244. [CrossRef]

34. Youssef, G.; Newacheck, S.; Yousuf, L.S. Insights into the displacement field in magnetoelectric composites. J. Intell. Mater. Syst. Struct. 2020, 31, 436-444. [CrossRef]

35. Bichurin, M.I.; Viehland, D. Magnetoelectricity in Composites; CRC Press: Boca Raton, FL, USA, 2011; ISBN 9789814267830.

36. Zheng, J.; Takahashi, S.; Yoshikawa, S.; Uchino, K.; De Vries, H. Heat Generation in Multilayer Piezoelectric Actuators. J. Am. Ceram. Soc. J. Amer. Ceram. Soc. 1996, 79, 3193-3198. [CrossRef] 